\title{
BMJ Open Cross-sectoral communication by bringing together patient with cancer, general practitioner and oncologist in a video-based consultation: a qualitative study of oncologists' and nurse specialists' perspectives
}

\author{
Dorte Gilså Hansen (D) , ,2,3 Theis Bitz Trabjerg (D) , ${ }^{1}$ Jeffrey James Sisler, ${ }^{4}$ \\ Jens Søndergaard (D) , ${ }^{1}$ Lars Henrik Jensen (D) $3,5,6$
}

To cite: Hansen DG,

Trabjerg TB, Sisler JJ, et al. Cross-sectoral communication by bringing together patient with cancer, general practitioner and oncologist in a video-based consultation: a qualitative study of oncologists' and nurse specialists' perspectives. BMJ Open 2021;11:e043038. doi:10.1136/ bmjopen-2020-043038

- Prepublication history and additional supplemental material for this paper are available online. To view these files, please visit the journal online (http://dx.doi.org/10.1136/ bmjopen-2020-043038)

Received 27 July 2020 Revised 22 February 2021 Accepted 16 April 2021

\section{Check for updates}

(c) Author(s) (or their employer(s)) 2021. Re-use permitted under CC BY-NC. No commercial re-use. See rights and permissions. Published by BMJ.

For numbered affiliations see end of article.

\section{Correspondence to}

Ass. professor Dorte Gilså

Hansen;

dorte.gilsa.hansen@rsyd.dk

\section{ABSTRACT}

Shared care models in the field of cancer aim to improve care coordination, role clarification and patient satisfaction. Cross-sectoral communication is pivotal. Involvement of patients may add to intended mechanisms.

A randomised controlled trial 'The Partnership Study' tested the effect of bringing together patient, general practitioner (GP) and oncologist for a consultation conducted by video.

Purpose As part of the process evaluation, this study aimed to explore experiences, attitudes and perspectives of the oncological department on sharing patient consultations with GPs using video.

Methods Four semistructured interviews with five oncologists and four nurse specialists were conducted in February 2020. We focused on the informants' experiences and reflections on the potential of future implementation of the concept 'inviting the GP for a shared consultation by video'. The analyses were based on an inductive, openminded, hermeneutic phenomenological approach.

Results A total of six overall themes were identified: structuring consultation and communication, perceptions of GP involvement in cancer care, stressors, making a difference, alternative ways of cross-sector communication and needs for redesigning the model. The concept made sense and was deemed useful, but solving the many technical and organisational problems is pivotal. Case-specific tasks and relational issues were targeted by pragmatically rethinking protocol expectations and the usual way of communication and structuring patient encounters. Case selection was discussed as one way of maturing the concept.

Conclusion This Danish study adds new insight into understanding different aspects of the process, causal mechanisms as well as the potential of future implementation of video-based tripartite encounters. Beyond solving the technical problems, case selection and organisational issues are important. Acknowledging the disruption of the usual workflow, the introduction of new phases of the usual encounter and the variety of patient-GP relationships to be embraced may help to
Strengths and limitations of this study

- Process evaluation of a randomised controlled trial is key to understanding intervention mechanisms

- Results may apply to other areas of shared care.

- Knowledge on tripartite video consultations is sparse.

- Informants were restricted to the oncological team.

Some perspectives may have been overlooked.

better understand and comply with barriers and facilitators of communication and sharing.

Trial registration number NCT02716168.

\section{BACKGROUND}

Continuity of care is a huge challenge for all healthcare systems as well as for many patients with chronic disorders like cancer. ${ }^{1}$ In periods with intensive treatment in hospital, it is often unclear to patients who will take care of their comorbidities and psychosocial aspects of living with a life-threatening disorder. ${ }^{2}$ The integration of general practitioners (GPs) into survivorship care is needed and underpinned internationally. ${ }^{3}$ However, GPs may feel insufficiently informed by specialists and thereby be left with an unclearly defined role. $^{45}$

Various shared care models have been developed in the field of cancer, primarily involving the GP and specialist in a formal explicit manner. ${ }^{6-8} \quad$ Communication between them through the exchange of information and arranging responsibility to improve the follow-up management has been in focus. ${ }^{7}$ Formal communication 
channels have been defined by discharge letters, cancer care plans, electronic platforms for text messaging or phone calls from nurse specialists. ${ }^{9-11}$ Reviews have concluded that these different models enable GPs' involvement in survivorship care and help the cooperation between the hospital and primary care. ${ }^{781112}$ However, patients themselves have only been scarcely involved, and no significant improvement in quality of life has been observed. ${ }^{78}$

During the last decade, there has been increasing attention to patient involvement and shared decision making in clinical oncology. Studies have stressed the importance of patient-centred communication, preparing patients for self-management and knowing who is going to help with what aspects of future treatment. ${ }^{1314}$ Having patient-centeredness as a key value of healthcare, we developed a shared care model based on a formal way of cross-sector communication giving room for engagement of the patient. ${ }^{15}$ Being aware of geographical constraints, but appreciating the living dialogue and seeing each other, we designed an intervention bringing patient with cancer, GP and oncologist together in a virtual room using video. ${ }^{16} 17$ Oncologists invite GPs to participate in usual patient encounters, so that they may share knowledge and divide on responsibility and specific tasks during treatment and survivorship. A randomised controlled trial (RCT) has been carried out to test this intervention. ${ }^{16}$

As outlined in the Medical Research Council Guidelines for complex interventions, process evaluation is an important part of trial evaluation. ${ }^{18}$ To fully understand trial mechanisms, acceptability, user perspectives and clues for future implementation strategies, tailored research should capture what was delivered and understand complex pathways. ${ }^{19}$

\section{AIMS}

As part of the process evaluation of the Partnership Study ${ }^{16}$ this paper aimed to explore experiences, attitudes and perspectives of doctors and nurses working in clinical oncology on sharing patient consultations with GPs using video.

\section{MATERIAL AND METHODS}

The empirical data were sampled by interviewing oncologists and specialist nurses who have participated in the tripartite consultations carried out as part of the 'Partnership Study'.

\section{The Partnership Study}

The design of the RCT has been described in detail elsewhere. ${ }^{16}$ To put it briefly, GPs of the 140 adult patients with cancer allocated to the intervention arm were invited by the oncological department at Vejle Hospital to one of the consultations during treatment with chemotherapy.
Box 1 Consultation guide including a list of potential themes presented for the general practitioners and oncologists before the partnership consultation

Consultation structure, aim and content

- The oncologist acts as chair of the shared video consultation.

- The duration of the consultation should be 10-20 min.

- The oncologist started by introducing the participants and the purpose of the shared consultation:

- Exchange of information between all participants for the benefit of the patient.

- Role and tasks clarification between the oncology department and the general practitioner.

- The consultations conclude with a summary where it is clarified whether a follow-up is needed at the general practitioner or oncology department.

- The consultation and its agreements are documented in the hospital electronic patient record, sent to the general practitioner and made available for the patient.

Potential themes for the consultation:

- A summary of the patient trajectory.

- Patient concerns and desire for the consultation.

- Sharing knowledge regarding comorbidity.

- Psychosocial resources and needs.

- Agreements on who should take care of what and when in the future.

- Physical well-being.

- Medicine.

Psychological well-being.

- Relatives.

- Ability to work.

- Late complications and side effects of the treatment.

$>$ Other.

The patients could choose to sit by the oncologist or by the GP, the latter chosen by one-fifth.

The overall aim of the trial was to assess the impact of the shared consultation on patient-perceived intersectoral cooperation and continuity of care, cancer-related distress and health-related quality of life. Based on a patient-centred approach, sharing a consultation with the two professionals should help to clarify the roles and tasks of the GP and oncologist, respectively, thereby improving patient comfortability with future treatment. At the same time, professionals could share relevant knowledge. Before each consultation, the oncologist and GP were handed a short consultation guide informing about consultation structure, aim and potential themes (box 1).

Due to 21 GPs' declination to participate, patient death and cancer severity, 79 consultations were conducted by 14 doctors (12 oncology specialists and 2 trainees). According to department routines, they were assisted by a nurse specialist. An unknown number of nurse specialists were involved in patient enrolment and the consultations.

As usual, patients were invited to bring along relatives.

\section{Participant recruitment}

Local key informants from the department were invited by LHJ (doctors) and a team-leading nurse specialist (nurses). 
Informants were selected purposively to be representative regarding gender and the disease-specific oncological teams.

\section{Data sampling}

Shortly after the last partnership intervention had taken place, DGH conducted four semistructured focus group interviews with five oncologists and four specialist nurses at Vejle Hospital. It was in February 2020, just before the COVID-19 pandemic reached Denmark. DGH introduced herself as $\mathrm{MD}$ with a long research career within family medicine and cancer, principal supervisor for PhD student TBT and deeply involved in the project. Coffee and small talk were shared to establish a comfortable environment. The participants were encouraged to speak freely and honestly. The conversation was based on the predetermined open-ended questions of the interview guide and other issues emerging from the dialogue $e^{20}$ (online supplemental file 1). The interview guide was defined by DGH and JS. Trained in focus group interviewing, DGH simultaneously observed the interaction dynamics within the groups. ${ }^{21}$ Due to practical reasons and considerations about the hierarchical nature of participants, oncologists and nurse specialists were grouped separately. ${ }^{20} 22$ None besides interviewer and informants were present.

As planned, the interviews took $45-55 \mathrm{~min}$. No repeat interviews were carried out. The preliminary data analysis coincided with data collection.

The interviews were audiotaped and transcribed verbatim by a trained research assistant. ${ }^{23}$ Transcripts were not returned to informants for comments.

\section{Further on researcher characteristics and reflexivity}

Trial ownership, workplace relationship with informants, medical specialisation and hierarchical structures may be potential sources of bias. All authors are owners of the trial and have been deeply involved in planning and implementation of the RCT: DGH with the role as main supervisor for PhD student TBT, and LHJ as chief specialist at the oncological department. In these roles, LHJ and TBT facilitated professional engagement, patient enrolment and technical issues. The professors JS and JJS have a long clinical career in family medicine and cancer research. During the analytical phase, we repeatedly reflected on these potential sources of bias.

\section{The process of analysing and reporting}

The analyses were based on an inductive, hermeneutic phenomenological approach. ${ }^{23}{ }^{24}$ After each interview, DGH familiarised with data by listening reflectively to the audio recordings and making interpretive notes. Themes, patterns and codes emerged. She continued with a careful reading of the transcripts, iteratively defining and organising codes into meaning-bearing entities. This data managing was supported by the software NVivo V.12. Based on manuscript drafts, themes, codes and meanings were discussed orally and by email with coauthors resulting in minor reorganisation and rephrasing. Subsequently, the number of quotations was reduced to onethird. Half-finished sentences and irrelevant wording were removed and replaced by '[...]'. Finally, DGH checked the grounding of the results in the empirical material. ${ }^{21}$

In the double role of coauthor and informant, LHJ did the member checking. All coauthors were involved in study designing and gave critical comments to manuscripts.

The reporting of the study follows the Standards for Reporting Qualitative Research (SRQR) ${ }^{25}$ and includes the Consolidated criteria for Reporting Qualitative research checklist (online supplemental file 2).

\section{Patient and public involvement}

Representatives from the Patient and Relatives Council at the hospital took part in unfolding the conceptual idea of the intervention, were involved in the pilot study including revision of questionnaires (content, way of administration and layout) and revision of intervention components. Furthermore, a workshop with the Patient and Relatives Council is planned when the final results from the RCT are ready (late 2021).

\section{RESULTS}

The length of the interviews was regarded as adequate. A relaxed atmosphere and good group dynamic were reached giving room for synergistic thoughts and exchange of views.

Thoughts pop up in your mind when you start talking about it. Lots of ideas. (Specialist nurse)

In most cases, the informants referred to cases with the patient placed at the oncological department. During the analysis, 15 categories and 6 overall themes emerged (table 1).

\section{Theme 1: structuring the consultation and communication Tailoring to individual tasks}

The oncologists remembered the consultation guide. However, they had quickly 'found their own way' of conducting these consultations and pragmatically tailored protocol expectations to organisational issues and specific tasks. Some oncologists preferred to explain topics as CT scans to patients without having the GP onboard. Private prediscussions and postdiscussions with the patient were thereby introduced:

In case there was a scan result I discussed it with them [patient and relatives] before, so they were not - so to speak - not taken hostage in the conversation, [...] and then after a short time I call the GP; and then finish afterward, conclude with a summary and by asking if there is anything I should know when their GP is not there. (Oncologist)

\section{Rethinking the usual encounter}

From the department perspective, a partnership consultation with the patient placed at the department was framed with three to five phases: the oncologist: (1) had 
Table 1 Overall themes and categories defined by the analysis of focus group interview with oncologists and nurse specialist experienced with the partnership intervention bringing together patient, GP and oncologist for a consultation shared by video

\begin{tabular}{|c|c|}
\hline Overall themes & Categories \\
\hline $\begin{array}{l}\text { Structuring consultation and } \\
\text { communication }\end{array}$ & $\begin{array}{l}\text { Tailoring to individual tasks. } \\
\text { Rethinking the usual } \\
\text { encounter. }\end{array}$ \\
\hline $\begin{array}{l}\text { Perceptions of GP } \\
\text { involvement in cancer care }\end{array}$ & $\begin{array}{l}\text { The patient-GP relationship. } \\
\text { GP behaviour. } \\
\text { Premises for the consultation. }\end{array}$ \\
\hline Stressors & $\begin{array}{l}\text { Technical stressors. } \\
\text { Organisational stressors. } \\
\text { Case-related stressors. }\end{array}$ \\
\hline Making a difference & $\begin{array}{l}\text { At group level. } \\
\text { At the individual level. }\end{array}$ \\
\hline $\begin{array}{l}\text { Alternative ways of cross- } \\
\text { sector communication }\end{array}$ & $\begin{array}{l}\text { Workshops. } \\
\text { Phone calls. } \\
\text { Discharge letters. }\end{array}$ \\
\hline $\begin{array}{l}\text { Needs for redesigning the } \\
\text { model }\end{array}$ & $\begin{array}{l}\text { Urgent prerequisites. } \\
\text { Wanted changes. }\end{array}$ \\
\hline
\end{tabular}

GP, general practitioner.

a short dialogue with the specialist nurse, (2) included patient and relatives, (3) continued with the shared part of the consultation having the GP on board, (4) finished having some extra time with patient and relative and (5) left the room leaving the patient and relative together with the specialist nurse. In case the patient was sitting by the GP, only phases 1 and 3 came into play, eventually finished with a short dialogue between oncologist and nurse specialist.

All informants agreed that the oncologist had been the leader of the consultation and that the patient was in focus. To a varying degree, the GPs 'took their time', often in the second half of the consultation. Also remembered were situations where the GP partly took over:

He [the GP] talked a lot with the patient. I was sidelined, but, but I don't think it mattered. (Oncologist)

Regarding communication, additional efforts were put into addressing the different interests, expectations and premises that patients and GPs represented:

$[\ldots]$ in a situation like this you make much of an effort to be - even more - pedagogical, for both the patient and the GP. (Oncologist)

\section{Theme 2: perceptions of GP involvement in cancer care} The patient-GP relationship

A variety of patient-GP relationships were to be embraced. Some patients had clearly explained their relational situation beforehand. Others did so during study enrolment or the introductory phase of the consultation. In addition to well-established professional relations, the informants told of patients that had not been in contact with their GP during the cancer treatment or even since long before diagnosis:

If you don't have a good relation with your doctor, if you don't know ... or haven't visited much [...]. In some way, it is a stranger sitting by. (Specialist nurse)

Some patients distrusted their GP due to a diagnostic phase that in their opinion had been too long or included misunderstandings. The oncologists thought those patients had denied participating or their GP had. However, the specialist nurses told them they had thoroughly recommended them to participate and were impressed by their courage to participate. They were very aware of challenges and tensions during these consultations.

\section{GP behaviour}

The large heterogeneity in GPs' behaviour and in patients' perception of the GPs' role was discussed. Some GPs saw the patient regularly. Other GPs had given the patient the impression of being unwilling 'to take part' and 'take on responsibility' during the oncological treatment, for example, of pain and treatment-related hypertension.

What does it mean when the patient says, "my GP is not willing to help me with this'? In fact, I don't think it is true. (Oncologist)

\section{Premises for the consultation}

Thus, from time to time, complex aspects of the patientGP relationship were premises for the consultations. Although not always understanding the relational tensions and what was meant by the GPs, attention was paid to barriers and facilitators to the communication.

\section{Theme 3: stressors}

All informants were concerned about the stress by the inclusion of video and the GP as a third party.

\section{Technical stressors}

Technical issues related to the video connection were time consuming, frustrating, affecting their professionalism and sometimes unsolvable.

[...] consultations went wrong. You know, we failed to get in contact at all. I also had some ...we ended up calling by phone and talked without video [...] so frustrating $[\ldots]$ you get behind schedule ... knowing that somebody is at the other end, waiting, and wasting time, and the patient is just sitting, looking. It is incredibly unprofessional. (Oncologist)

\section{Organisational stressors}

The informants all recognised tight ambulatory schedules and being routinely behind time. A partnership consultation was a clinical obligation, time-fixed like 
multidisciplinary team conferences or encounters using external interpreting services. However, knowing that an external colleague was expecting them at a specific time was perceived as extraordinarily stressful.

The informants were used to encounters partly defined by protocols. Integrating too many things and concepts into one encounter could be challenging and set participants under pressure. However, beyond the critical technical issues, the extra topics of a partnership intervention were acceptable, and the typical length of 12-14 min was referred to as appropriate.

Both internal and external organisational stressors had the potential of compromising the conceptual idea of the partnership consultation. The GP and his or her level of calmness, engagement or irritation was mentioned. However, using a lot of effort, they typically succeeded to overcome starting problems.

\section{Case-related stressors}

Case-related stressing factors included situations where the oncologist experienced no apparent need for having the GP on board: low-complex cases like otherwise healthy women with breast cancer and patients already well supported by the GP. These situations were by some informants perceived a wrong prioritisation of their professional time.

In case the patient has no problems, the aim of the consultation becomes somewhat forced. (Oncologist)

Especially if a difficult message had to be communicated, it called for extra attention when having a third part in the room:

In case you are focusing on the patient when breaking bad news, it is difficult to keep an eye on the GP, too. (Oncologist)

\section{Theme 4: making a difference}

The partnership interventions were deemed useful in several ways regarding individual trajectories. Furthermore, at a group level, they had the potential to make a difference for future patients. Getting more insight into GPs' competences and areas of concerns by meeting them in action changed habits and professional persuasions:

I have become aware of $[\ldots]$ how much they [the GPs] engage, counsel, and support. I have seen some very skilled and very empathic GPs [...]. Previously, I could not advise the patients about that. I quickly said 'let us ask the palliative team'. (Specialist nurse)

Previously, when I said 'try consulting your GP', I felt as if I pushed it out of my mind, but now... I say so because... it will work out better [...]. It is not a bad solution, or because I am lazy. (Oncologist)

Several examples were given regarding how a partnership consultation was perceived as useful to the patient. Being together paved the way for overcoming mistrust. Allowing the GP to empathetically welcome the patient and relatives for psychosocial and palliative care and underline their role and comfortability treating comorbidities, cancer pain, etc, were other examples. However, to what degree the partnership consultation had been pivotal to the informants was more unclear. In many cases, relationships were long established, and things were organised beforehand.

A few extraordinary examples were remembered like one with a drug-addicted patient. According to the oncologist, sharing that dialogue about future prescribing of pain killers was very meaningful and would never have happened without this project.

Completing a long-lasting trajectory was easier for both patient and oncologist when convinced about the GP's role, readiness and competences. Partnership consultations thus provided a measure of relief to the oncologist.

With the exception of one oncologist, the informants broadly agreed that having the GP on board a patient consultation using video was worth implementing in routine clinical practice-in a structured way.

A scheduled appointment. I know that the doctor at the other end has time and that I have time. You see, that is perfect. I think we talk way too little with the GPs. Way too little. (Oncologist)

\section{Clues to implementation}

Before discussing the need for redesigning the concept, the oncologists challenged alternative models of communication and partnering with GPs. However, all compromised the conceptual idea of patient-centeredness.

\section{Theme 5: alternative ways of cross-sectoral communication}

Workshops for GPs were mentioned as one way of partnering at a general level. Calling the GP by phone was discussed as a more flexible way of professional sharing regarding individual patients. Relating to the perceived relevance of what was talked about during partnership consultations, an oncologist criticised their discharge summaries to GPs. Laughing, the group agreed to them being useless alternatives:

Discharge letters saying 'the patient was here for chemotherapy and we gave so-and-so many milligrams of these seven drugs' [...] they don't give a damn [...] what is important to them, is what we talk about in consultations like these [...] We would never mention chemo doses in a dialogue like this. (Oncologist)

\section{Theme 6: needs for redesigning the model}

First, overcoming all the technical problems was an urgent prerequisite for implementation. Second, selection of the right patients was underlined. Bypassing lowcomplexity cases and those already treated well by their GP were proposed but not agreed on. Several examples of 'the right time' for a partnership consultation were mentioned: in case the patient needs to re-establish the contact to and trust in their GP, if the GP is in doubt 
about his or her role and expertise and has questions to the oncologist, if a handover of the patient and responsibility is to take place, if the patient is in a vulnerable situation waiting for the blood counts to come into level before starting treatment, and obviously, if the patient is severely ill by cancer or complex comorbidity. Furthermore, scheduling these consultations at the beginning of a day was mentioned as a way of easing the challenge of two professionals being ready at the same time.

\section{DISCUSSION}

This study explored oncologists' and specialist nurses' experiences and perspectives of a new way of crosssector communication established by inviting GPs by video for patient encounters. The concept was deemed feasible and useful but gave rise to several challenges. As patients do not always have a supportive experience of their GP, a variety of patient-GP relationships were to be embraced professionally. Case-specific tasks and organisational stressors were targeted by pragmatically rethinking protocol expectations, and flow and communication of usual encounters. Centred around 12-15 min shared by video, partnership consultations included up to five phases. Technical problems challenged professional roles. Case selection was discussed as one way of maturing the concept for future use.

\section{Strengths and limitations}

We aimed to explore the perspectives of the oncology team only, either patient, relative or GP perspectives. Inviting both cancer specialist physicians and nurses for interview maximised the richness of our data. The sample succeeded in having male and female oncologists representing all disease-specific teams. The female specialist nurses expressed the gender representation at this position but not all teams. The informants' level of experience with partnership consultations varied giving room for yet another variation. The interviewer managed to reach a relaxed atmosphere and a good group dynamic. However, being deeply involved in the RCT and having the local project leader (LHJ) as one of the informants could have compromised openness. ${ }^{22}$ Meanwhile, a range of experiences and perspectives were uncovered including critical statements. Each member had time to voice their views and the themes of the interview guide were covered. However, we may not be sure if we reached saturation. ${ }^{22}$ It was deemed fruitful for the analytical process that the authors represented a wide variety of medical and research experiences and actively reflected on their positions.

When interpreting the results, we need to be aware that selection processes left out experiences with patients and GPs unwilling to take part in the RCT, for example, some with strained relationships or patients feeling weak. Furthermore, Vejle Hospital is known for being innovative and open-minded regarding cross-sectoral cooperation, shared decision making ${ }^{15}$ and patient-centred communication. ${ }^{26}$ In more conservative hospitals, resistance to this type of innovation may be larger.

\section{Discussion of results}

By studying records of partnership interventions, we have previously shown that these consultations succeeded in being patient centred and supported the building of relationship between patient and GP, patient and oncologist, as well as between the two healthcare providers. ${ }^{27}$ Interviewing oncologists and specialist nurses added to the empirical evidence and process evaluation of the trial. Patients do not necessarily trust or know their GP, meaning that lack of confidence in the patient-GP relation is a potential challenge for these and similar tripartite consultations. A review has shown the significance of relationship and communication for patient satisfaction in oncology. ${ }^{28}$ Studies of video consultations have shown that the patients who benefit the most from this format are those with a good relation. ${ }^{29}{ }^{30}$ Including an extra health professional into the encounter meant embracing an unknown medical colleague as well as another professional relation, potentially closer, but sometimes tense or unfamiliar compared with their association with the patient. A review of the effectiveness of shared care models in cancer showing no effect on health outcomes argued that lack of confidence in primary care could have been an important confounding factor. ${ }^{7}$ Patients may stop seeing their GP when mistrusting their competences in cancer. ${ }^{31}{ }^{32}$ Furthermore, inspired by communication guidelines for medical encounters, we suggest that articulation of the character of the patient-GP relation before and during the opening of a tripartite consultation may improve the atmosphere, patient satisfaction and health outcomes. ${ }^{28} 33$ Our finding challenges the general assumption of healthcare systems based on comprehensive primary care: patients have a family physician who they know well from the treatment of their illness, comorbidities and trivialities. ${ }^{34}$ Care delivery through multidisciplinary teams in general practice may partly explain our observation. ${ }^{335}$ Overlooking or neglecting the variety of patient-GP relationships may thus compromise the potential of future shared care strategies.

The literature on video consultations with patients at home have concluded that technical problems disrupt workflow, consultation process, and as highlighted by our informants, affect professional roles. ${ }^{9} 303637$ The need for a reliable, easy-to-use system before translation into clinical practice is indisputable. ${ }^{30}$ The COVID-19 pandemic has sped up the integration of video consultations in healthcare. New technologies are developed and much is learnt. ${ }^{36}$ We suggest that also cross-sectoral use has become less discouraging to patients and professionals and even more relevant. To ensure user acceptability and system stability, we highly recommend exhaustive pilottesting in the actual setting. ${ }^{18}$

The delay that often arise during working days was perceived more burdensome than usual knowing that an external medical specialist would need to wait. A virtual 
waiting room has been introduced for patients waiting for online consultations but may not fully relieve specialists waiting for a tripartite consultation. ${ }^{29}$ However, optimising the concept by scheduling in early working hours may improve professional acceptability and reduce wasted time and stress levels.

Several studies have underlined the willingness of GPs to provide cancer care, but their professional confidence varies in line with the level of information and role clarification. ${ }^{49}$ The latter are consistently deemed necessary for care coordination by both primary and secondary care professionals. ${ }^{4041}$ We have previously shown that the participating physicians feel ready to unravel misconceptions, listen and support each other. ${ }^{27}$ From the present study, new potential mechanisms of cross-sectoral consultations emerged. For the oncologists, bringing together was regarded as a way to come to understand patients' perception of being declined cancer-related support from their GP.

\section{Perspectives for practice and research}

In addition to planned studies of the effectiveness of the partnership intervention and previous process evaluations, ${ }^{162742}$ this study adds important knowledge regarding fidelity, acceptability, perceived usefulness and needs for a redesign for future use. ${ }^{19}$ This new tool for communication and partnering disrupts the existing workflow and challenges the consultation structure, communication and interpersonal relationship of existing oncological encounters. Acknowledging the variety of patient-GP relationships to be embraced and the introduction of new phases of the usual encounter may help comply with barriers and facilitators of the concept. Case selection seems appealing for future models. ${ }^{43}$

\section{Author affiliations}

${ }^{1}$ Research Unit for General Practice, Faculty of Health Sciences, University of Southern Denmark, Odense, Denmark

${ }^{2}$ Center for Shared Decision Making, Lillebælt Hospital, University Hospital of Southern Denmark, Vejle, Denmark

${ }^{3}$ Institute of Regional Health Research, University of Southern Denmark, Vejle, Denmark

${ }^{4}$ Department of Family Medicine, University of Manitoba Faculty of Health Sciences, Winnipeg, Manitoba, Canada

${ }^{5}$ Department of Oncology, Lillebælt Hospital, University Hospital of Southern Denmark, Vejle, Denmark

${ }^{6}$ Danish Colorectal Cancer Center South, Center of Clinical Excellence, Lillebaelt Hospital, University Hospital of Southern Denmark, University of Southern Denmark Vejle, Denmark

Acknowledgements We are thankful to all patients and professionals who contributed to the Partnership Project and especially to oncologists and nurse specialists participating in the focus group interviews. We furthermore thank research assistant Alexander Gilså Hansen for the transcription of audiotapes and proofreading of the manuscript.

Contributors All authors took part in the initiation and design of this study, the interpretation of data and the critical revision of the manuscript for important intellectual content. LHJ and DGH recruited the study participants. DGH made all interviews and had full access to all the data in the study and took responsibility for the integrity of the data and the accuracy of the data analysis. DGH drafted the manuscript. All authors have been involved in all phases of the randomised controlled trial, which this study is part of. TBT took lead on most practical and technical issues related to the intervention. TBT and LHJ promoted the trial at the department. DGH was the main supervisor for PhD student TBT.

Funding The Partnership Project was financially supported by the Danish Cancer Society (R156-A10018-16-S49); The Region of Southern Denmark Research Foundation; the Region of Southern Denmark PhD Foundation; Lillebaelt Hospital, University Hospital of Southern Denmark; University of Southern Denmark; The Foundation for General Practice (A1850); and the ML Jørgensen and Gunnar Hansen Foundation (ID 2956). The two last-mentioned has funded this part of the study.

Competing interests None declared.

Patient consent for publication Not required.

Ethics approval At the time of oral invitation for an interview, all informants knew the partnership intervention in detail from their clinical work. At the interview, all gave written informed consent for research use of the information shared during the interview. According to EU and national Data Protection Regulations, the study was approved by the University of Southern Denmark Research \& Advice Organisation, registration number 10.902 .

Provenance and peer review Not commissioned; externally peer reviewed.

Data availability statement No data are available. Unfortunately, we are unable to share data, as we are limited under data sharing agreements that do not allow us to share the vendor data with third parties.

Supplemental material This content has been supplied by the author(s). It has not been vetted by BMJ Publishing Group Limited (BMJ) and may not have been peer-reviewed. Any opinions or recommendations discussed are solely those of the author(s) and are not endorsed by BMJ. BMJ disclaims all liability and responsibility arising from any reliance placed on the content. Where the content includes any translated material, BMJ does not warrant the accuracy and reliability of the translations (including but not limited to local regulations, clinical guidelines, terminology, drug names and drug dosages), and is not responsible for any error and/or omissions arising from translation and adaptation or otherwise.

Open access This is an open access article distributed in accordance with the Creative Commons Attribution Non Commercial (CC BY-NC 4.0) license, which permits others to distribute, remix, adapt, build upon this work non-commercially, and license their derivative works on different terms, provided the original work is properly cited, appropriate credit is given, any changes made indicated, and the use is non-commercial. See: http://creativecommons.org/licenses/by-nc/4.0/.

\section{ORCID iDs}

Dorte Gilså Hansen http://orcid.org/0000-0002-5946-9968

Theis Bitz Trabjerg http://orcid.org/0000-0002-7632-8227

Jens Søndergaard http://orcid.org/0000-0002-1629-1864

Lars Henrik Jensen http://orcid.org/0000-0002-0020-1537

\section{REFERENCES}

1 Haggerty JL, Roberge D, Freeman GK, et al. Experienced continuity of care when patients see multiple clinicians: a qualitative metasummary. The Annals of Family Medicine 2013;11:262-71.

2 Grunfeld E. The two solitudes of primary care and cancer specialist care: is there a bridge? Curr Oncol 2017;24:69-70.

3 Rubin G, Berendsen A, Crawford SM, et al. The expanding role of primary care in cancer control. Lancet Oncol 2015;16:1231-72.

4 Lawrence RA, McLoone JK, Wakefield CE, et al. Primary care physicians' perspectives of their role in cancer care: a systematic review. J Gen Intern Med 2016;31:1222-36.

5 Dossett LA, Hudson JN, Morris AM, et al. The primary care provider (PCP)-cancer specialist relationship: A systematic review and mixedmethods meta-synthesis. CA Cancer J Clin 2017;67:156-69.

6 Tomasone JR, Vukmirovic M, Brouwers MC, et al. Challenges and insights in implementing coordinated care between oncology and primary care providers: a Canadian perspective. Curr Oncol 2017:24:120-3.

7 Zhao Y, Brettle A, Qiu L. The effectiveness of shared care in cancer Survivors-A systematic review. Int J Integr Care 2018;18:2.

8 Perfors IAA, May AM, Boeijen JA, et al. Involving the general practitioner during curative cancer treatment: a systematic review of health care interventions. BMJ Open 2019;9:e026383.

9 Voruganti T, Grunfeld E, Makuwaza T, et al. Web-Based tools for Text-Based patient-provider communication in chronic conditions: Scoping review. J Med Internet Res 2017;19:e366.

10 Bergholdt SH, Larsen PV, Kragstrup J, et al. Enhanced involvement of general practitioners in cancer rehabilitation: a randomised controlled trial. BMJ Open 2012;2:e000764. 
11 Brennan ME, Gormally JF, Butow P, et al. Survivorship care plans in cancer: a systematic review of care plan outcomes. $\mathrm{Br} \mathrm{J}$ Cancer 2014;111:1899-908.

12 Tomasone JR, Brouwers MC, Vukmirovic M, et al. Interventions to improve care coordination between primary healthcare and oncology care providers: a systematic review. ESMO Open 2016;1:e000077.

13 Lawn S, Fallon-Ferguson J, Koczwara B. Shared care involving cancer specialists and primary care providers - What do cance survivors want? Health Expect 2017;20:1081-7.

14 Hong YA, Hossain MM, Chou W-YS. Digital interventions to facilitate patient-provider communication in cancer care: a systematic review. Psychooncology 2020;29:591-603.

15 Steffensen KD, Vinter M, Crüger D, et al. Lessons in integrating shared decision-making into cancer care. J Oncol Pract 2018;14:229-35.

16 Trabjerg TB, Jensen LH, Søndergaard J, et al. Improving continuity by bringing the cancer patient, general practitioner and oncologist together in a shared video-based consultation - protocol for a randomised controlled trial. BMC Fam Pract 2019;20:86.

17 Jess M, Timm H, Dieperink KB. Video consultations in palliative care: a systematic integrative review. Palliat Med 2019;33:942-58.

18 Craig P, Dieppe P, Macintyre S, et al. Developing and evaluating complex interventions: the new medical Research Council guidance. BMJ 2008;337:a1655.

19 Moore GF, Audrey S, Barker M, et al. Process evaluation of complex interventions: medical Research Council guidance. BMJ 2015;350:h1258

20 Malterud K. Theory and interpretation in qualitative studies from general practice: why and how? Scand J Public Health 2016;44:120-9.

$21 \mathrm{Cyr} \mathrm{J}$. The pitfalls and promise of focus groups as a data collection method. Sociol Methods Res 2016;45:231-59.

22 Vicsek L. Issues in the analysis of focus groups: Generalisability, Quantifiability, treatment of context and quotations. Qualitative Report 2010;15:122-41.

23 Moser A, Korstjens I. Series: practical guidance to qualitative research. Part 3: sampling, data collection and analysis. Eur J Gen Pract 2018;24:9-18.

24 Neubauer BE, Witkop CT, Varpio L. How phenomenology can help us learn from the experiences of others. Perspect Med Educ 2019;8:90-7.

25 O'Brien BC, Harris IB, Beckman TJ, et al. Standards for reporting qualitative research: a synthesis of recommendations. Acad Med 2014;89:1245-51.

26 Ammentorp J, Graugaard LT, Lau ME, et al. Mandatory communication training of all employees with patient contact. Patient Educ Couns 2014;95:429-32.

27 Trabjerg TB, Jensen LH, Sondergaard J, et al. Investigating whether shared video-based consultations with patients, oncologists, and GPs can benefit patient-centred cancer care: a qualitative study.
BJGP Open 2020;4. doi:10.3399/bjgpopen20X101023. [Epub ahead of print: 23 Jun 2020].

28 Prip A, Møller KA, Nielsen DL, et al. The Patient-Healthcare professional relationship and communication in the oncology outpatient setting: a systematic review. Cancer Nurs 2018;41:E11-22.

29 Greenhalgh T, Shaw S, Wherton J, et al. Real-World implementation of video outpatient consultations at macro, meso, and micro levels: Mixed-Method study. J Med Internet Res 2018;20:e150.

30 Donaghy E, Atherton H, Hammersley V, et al. Acceptability, benefits, and challenges of video consulting: a qualitative study in primary care. Br J Gen Pract 2019;69:e586-94.

31 Mikkelsen T, Sondergaard J, Sokolowski I, et al. Cancer survivors' rehabilitation needs in a primary health care context. Fam Pract 2009;26:221-30.

32 Larsen MB, Hansen RP, Olesen F, et al. Patients' confidence in their GP before and after being diagnosed with cancer. Br J Gen Pract 2011;61:e215-22.

33 Mitchell GK, Burridge LH, Colquist SP, et al. General practitioners' perceptions of their role in cancer care and factors which influence this role. Health Soc Care Community 2012;20:607-16.

34 Pedersen KM, Andersen JS, Søndergaard J. General practice and primary health care in Denmark. J Am Board Fam Med 2012;25 Suppl 1:S34-8.

35 Wright M, Mainous A. Can continuity of care in primary care be sustained in the modern health system? Aust J Gen Pract 2018;47:667-9.

36 Greenhalgh T, Wherton J, Shaw S. Video consultations for covid-19. An opportunity in a crisis. BMJ 2020;368:m998.

37 Funderskov KF, Raunkiær M, Danbjørg DB, et al. Experiences with video consultations in specialized palliative home-care: qualitative study of patient and relative perspectives. J Med Internet Res 2019;21:e10208.

38 Thornton J. Covid-19: how coronavirus will change the face of general practice forever. BMJ 2020;368:m1279.

39 Guassora AD, Jarlbaek L, Thorsen T. Preparing general practitioners to receive cancer patients following treatment in secondary care: a qualitative study. BMC Health Serv Res 2015;15:202.

40 Easley J, Miedema B, O'Brien MA, et al. The role of family physicians in cancer care: perspectives of primary and specialty care providers. Current Oncology 2017;24:75-80.

41 Easley J, Miedema B, Carroll JC, et al. Coordination of cancer care between family physicians and cancer specialists: importance of communication. Can Fam Physician 2016;62:e608-15.

42 Trabjerg TB, Jensen LH, Søndergaard J, et al. Cross-sectoral video consultations in cancer care: perspectives of cancer patients, oncologists and general practitioners. Support Care Cancer 2021;29:107-16.

43 Nekhlyudov L, Mollica MA, Jacobsen PB, et al. Developing a quality of cancer survivorship care framework: implications for clinical care, research, and policy. J Natl Cancer Inst 2019;111:1120-30. 